\title{
La auscultación pulmonar en el siglo 21
}

\section{Lung auscultation in the 21 th century}

\section{Francisca Bertrand Z. ${ }^{a}$, Dafne Segall K. ${ }^{a}$, Ignacio Sánchez D. ${ }^{b}$, Pablo Bertrand N. ${ }^{b}$}

alnterna, Facultad de Medicina, Pontificia Universidad Católica de Chile, Santiago, Chile.

bUnidad de Enfermedades Respiratorias Pediátricas, Facultad de Medicina, Pontificia Universidad Católica de Chile, Santiago, Chile.

Recibido: 3 de octubre de 2019; Aceptado: 8 de marzo de 2020

\section{¿Qué se sabe del tema que trata este estudio?}

La auscultación pulmonar de los ruidos respiratorios ha logrado una adecuada estandarización gracias a avances en el análisis computacional y estudios de concordancia que han simplificado la nomenclatura.

\begin{abstract}
¿Qué aporta este estudio a lo ya conocido?
Este estudio revisa el rendimiento de la auscultación pulmonar en los niños en base a estudios clínicos y considera el análisis computacional de los mismos como forma de obtener mayor beneficio de esta técnica.
\end{abstract}

\section{Resumen}

La auscultación pulmonar es parte fundamental del examen físico para el diagnóstico de las enfermedades respiratorias. La estandarización que ha alcanzado la nomenclatura de los ruidos respiratorios, sumado a los avances en el análisis computacional de los mismos, han permitido mejorar la utilidad de esta técnica. Sin embargo, el rendimiento de la auscultación pulmonar ha sido cuestionado por tener una concordancia variable entre profesionales de la salud. Aun cuando la incorporación de nuevas herramientas diagnósticas de imágenes y función pulmonar han revolucionado la precisión diagnóstica en enfermedades respiratorias, no existe tecnología que permita reemplazar la técnica de auscultación pulmonar para guiar el proceso diagnóstico. Por una parte, la auscultación pulmonar permite seleccionar a aquellos pacientes que se beneficiarán de una determinada técnica diagnóstica, se puede repetir cuantas veces sea necesario para tomar decisiones clínicas, y frecuentemente permite prescindir de exámenes adicionales que no siempre son fáciles de realizar o no se encuentran disponibles. En esta revisión se presenta el estado actual de la técnica de auscultación pulmonar y su rendimiento objetivo basado en la nomenclatura actual aceptada para los ruidos respiratorios, además de resumir la evidencia principal de estudios de concordancia de auscultación pediátrica y su análisis objetivo a través de nueva tecnología computacional.
Palabras clave: Auscultación pulmonar; ruidos respiratorios; examen físico; niños 


\begin{abstract}
Lung auscultation is an essential part of the physical examination for diagnosing respiratory diseases. The terminology standardization for lung sounds, in addition to advances in their analysis through new technologies, have improved the use of this technique. However, traditional auscultation has been questioned due to the limited concordance among health professionals. Despite the revolutionary use of new diagnostic tools of imaging and lung function tests allowing diagnostic accuracy in respiratory diseases, no technology can replace lung auscultation to guide the diagnostic process. Lung auscultation allows identifying those patients who may benefit from a specific test. Moreover, this technique can be performed many times to make clinical decisions, and often with no need forcomplicated and sometimes unavailable tests. This review describes the current state-of-the-art of lung auscultation and its efficacy based on the current respiratory sound terminology. In addition, it describes the main evidence on respiratory sound concordance studies among health professionals and its objective analysis through new technology.
\end{abstract}

\section{Keywords:}

Lung auscultation; respiratory sounds; physical examination; childhood

\section{Introducción}

Los avances en medicina permiten hoy delimitar con precisión la anatomía de las estructuras del tórax a través de la realización de una tomografía axial computada de alta resolución con poca o mínima radiación. De forma similar, hoy es posible mejorar nuestro entendimiento de la obstrucción de la vía aérea al realizar la técnica de oscilometría de impulsos para medir resistencia y reactancia del aparato respiratorio o valorar la magnitud de inflamación presente en la vía aérea mediante el uso de la técnica de medición de fracción exhalada de óxido nítrico; sin embargo, estas herramientas diagnósticas presentan un adecuado rendimiento solo cuando están apropiadamente guiadas por la historia clínica y el examen físico.

La auscultación pulmonar, como parte del examen físico del tórax, fue considerada útil desde los tiempos de Hipócrates, pero no floreció como técnica diagnóstica hasta que el médico francés René Laënnec describiera la utilización de un instrumento y la clasificación de los ruidos respiratorios que permitiría mejorar el proceso diagnóstico ${ }^{1-3}$. Luego de 200 años, la auscultación pulmonar ha sufrido modificaciones en la nomenclatura producto de la traducción a distintos idiomas y de la valoración de su rendimiento en el proceso diagnóstico, pero se mantiene como un pilar básico en el proceso diagnóstico.

La auscultación pulmonar permite evaluar los ruidos generados en la vía aérea a través del flujo del aire, que se manifiestan con una frecuencia y una amplitud determinada que se integra con otros elementos clínicos del examen físico ${ }^{4}$. Su utilidad radica en que es una técnica fácil de aplicar, que entrega información inmediata y dinámica. Su desventaja es que se trata de una técnica subjetiva que se ve influenciada por la colaboración del paciente y por el ruido ambiental. Se ha cuestionado el rendimiento de esta técnica, debido a que se ha reportado una pobre concordancia entre evaluadores frente a un mismo hallazgo auscultatorio ${ }^{5,6}$; sin embargo, también existe evidencia de buena concordancia en la auscultación de ruidos seleccionados como ocurre entre distintos profesionales que se evalúan con grabaciones o videos ${ }^{7-9}$.

El análisis computarizado de ruidos respiratorios podría aportar objetividad al momento de analizar ruidos respiratorios debido a la estandarización que presenta actualmente ${ }^{10,11}$. Esta técnica permite la descomposición de la señal en sus diferentes frecuencias para caracterizar con más precisión este tipo de señales ${ }^{12,13}$ y analizar así de forma más objetiva los ruidos respiratorios (figuras 1,2); sin embargo, la auscultación con equipos electrónicos todavía no han logrado filtrar los ruidos ambientales que interfieren con la señal digital y tampoco el análisis está disponible de forma inmediata para el diagnóstico clínico.

El objetivo de esta revisión es presentar el rendimiento de la auscultación pulmonar pediátrica por medio del análisis crítico de estudios de concordancia de auscultación y del análisis computacional de ruidos respiratorios.

\section{Técnica de auscultación}

La auscultación pulmonar en el niño obliga a realizar una cierta rutina que permita obtener información relevante de forma eficiente. Para esto es fundamental contar un fonendoscopio adecuado al tamaño del paciente, que disponga de membrana y campana para la mejor auscultación de ruidos graves o agudos ${ }^{14,15}$. La técnica debe ser sistemática y siempre de forma comparativa buscando una respiración eficiente y comenzando por las zonas críticas antes que el niño provoque llanto. Para esto, el operador debe hablar con un tono de voz amigable y monótona, intentar ganar la con- 
fianza con gestos amables dirigidos al niño y mover las manos suavemente para no llamar la atención. En esta interacción es útil ser lúdico. Si bien un niño con llanto puede ser más difícil de auscultar, el llanto puede

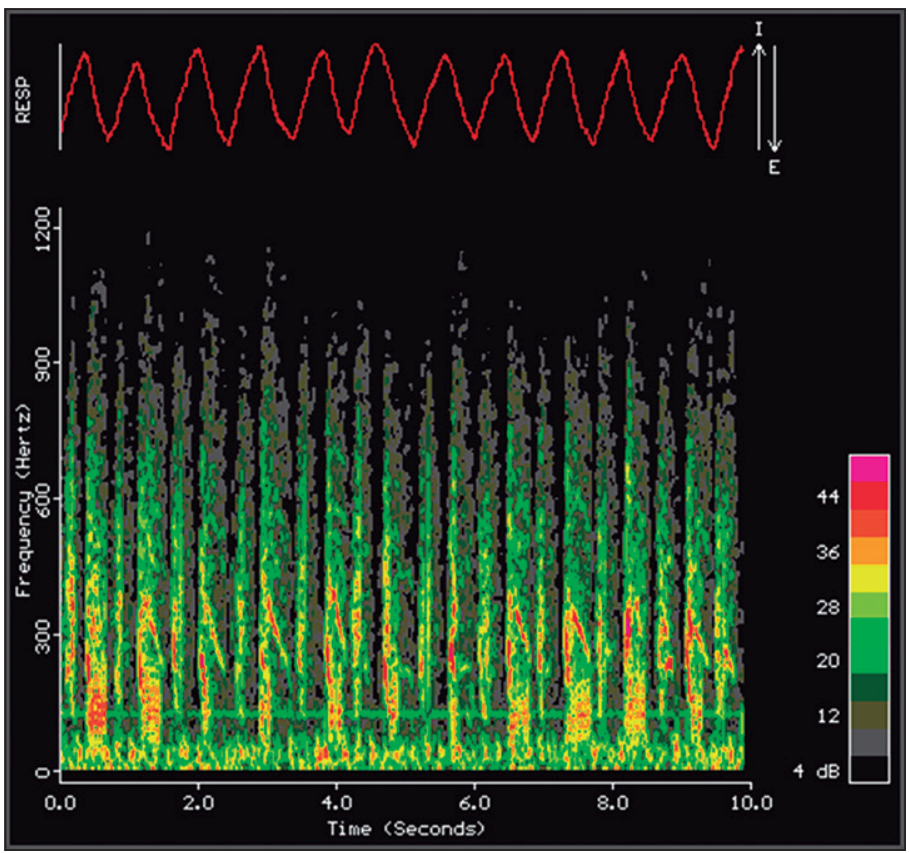

Figura 1. Respirosonograma de una grabación realizada en un lactante con bronquiolitis utilizando software de análisis RALE-Mediwave. Se puede apreciar flujo (curva en rojo), frecuencia ( $\mathrm{Hz}$ ) e intensidad (dB por colores) durante 10 segundos de grabación de ruidos pulmonares. Se logra visualizar en ambas fases de la respiración un amplio rango de frecuencia de ruidos respiratorios $(50$ a $800 \mathrm{~Hz}$ ), pero destaca mayor intensidad de frecuencia a nivel de 300 $\mathrm{Hz}$. Estos hallazgos verificados en el análisis de voltaje y frecuencia correspondientes corresponden a sibilancias. ser útil, ya que la respiración profunda que se produce entre cada sollozo a menudo intensifica los ruidos respiratorios. Por último, se debe considerar que la risa, la respiración forzada y la fonación generan ruidos artificiales que deben ser descartados apropiadamente $e^{15,16}$.

\section{Rendimiento de la auscultación}

La técnica de auscultación pulmonar ha sido evaluada de dos formas: por medio de la comparación de los hallazgos entre profesionales, y por medio de la valoración del rendimiento de un determinado ruido con un diagnóstico determinado.

La auscultación pulmonar es subjetiva, lo que ha obligado a evaluar el rendimiento de la discriminación de ruidos respiratorios a través de estudios de concordancia entre profesionales de la salud. Estos estudios han mostrado variabilidad de acuerdo al diseño utilizado. En un estudio de 180 niños que cursaban con bronquiolitis, Gajdos ${ }^{8}$ reportó una concordancia sustancial $(\kappa=0,73)$ inter-observador para la identificación de sibilancias entre distintos profesionales. En forma similar, Margolis ${ }^{17}$ reportó un resultado satisfactorio en un estudio realizado en 56 lactantes que se presentaron con sospecha de infección respiratoria baja donde la concordancia fue sustancial $(\kappa=0,63)$ para sibilancias espiratorias entre médicos. Esto se contrapone con el estudio de Bekhof ${ }^{18}$ realizado con la filmación de 27 niños mientras estaban en el servicio de urgencias, videos que fueron expuestos a enfermeras y médicos entrenados en la evaluación pediátrica de emergencia. La concordancia inter-observador para

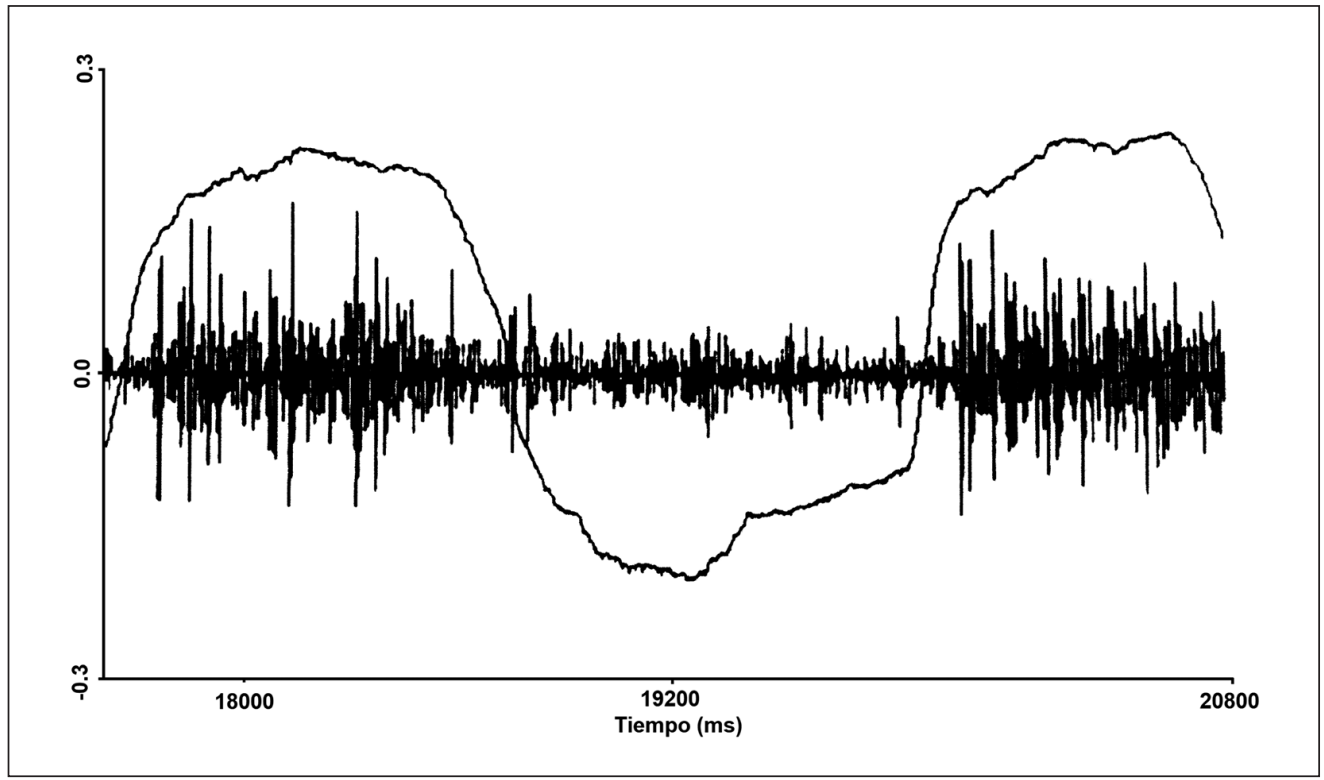

Figura 2. Diagrama de ruidos respiratorio donde se aprecia la señal acústica en relación con flujo respiratorio. Se logra apreciar onda sinusoidal tipo clásica compatible con sibilancias. 
sibilancias en este estudio fue baja $(\kappa=0,36)$ entre los distintos profesionales. De forma parecida, Jensen ${ }^{19}$ en 30 prematuros de edad gestacional 36-40 semanas encontró una concordancia inter-observador baja a media $(\kappa=0,34-0,54)$ entre médicos. Las razones que explican esta variabilidad obedecen a criterios de diseño. Como norma general, los estudios que presentan mejores resultados en concordancia entre profesionales resultan ser aquellos que han analizado pocas variables clínicas o han definido de forma mucho más exacta el ruido respiratorio a evaluar. Esto fue corroborado recientemente por el estudio de Melbye ${ }^{7}$ que utilizó 20 videos de pacientes reales y reportó una concordancia baja $(\kappa<0,4)$ para discriminación de ruidos adventicios entre médicos; sin embargo, la concordancia mejoró en forma significativa $(\kappa=0,59-0,62)$ cuando la clasificación de los ruidos respiratorios se simplificó en categorías inclusivas utilizando los mismos videos. De esta forma, estos estudios apoyan la idea que la simplificación de la nomenclatura y una buena definición de los términos mejoran la discriminación de los ruidos respiratorios entre profesionales.

Los estudios de concordancia entre profesionales también muestran distinto rendimiento dependiendo de los ruidos que son elegidos en el análisis. Muñoz $^{9}$ realizó un estudio de concordancia entre profesionales utilizando como referencia solo ruidos adventicios definidos en la nueva nomenclatura internacional de ruidos respiratorios ${ }^{20}$. La concordancia inter-observador reportada fue substancial pero variable $(\kappa=0,55-0,84)$ entre kinesiólogos del área respiratoria. Al analizar por separado los ruidos respiratorios, es notorio que la concordancia fue superior para las presencia de crepitaciones finas $(\kappa=0,84)$ comparada con la presencia de crepitaciones gruesas $(\kappa=0,69)$. Esto se podría explicar en base al rango de frecuencia que presentan los distintos ruidos ${ }^{21}$, siendo muy diferente del rango de frecuencia que presenta el murmullo pulmonar normal comparado con las crepitaciones finas. De este modo, es teóricamente más fácil para el profesional distinguir la presencia de crepitaciones finas que las crepitaciones gruesas.

La experiencia clínica ha sido uno de los factores que se consideró importante para explicar la concordancia entre evaluadores en la discriminación de ruidos respiratorios, pero los estudios que han buscado evaluar esta asociación no han encontrado diferencias entre grupos ${ }^{9,22}$. Al contrario, el estudio realizado por Muñoz ${ }^{9}$ reportó una relación inversa entre los años de experiencia y la discriminación de estridor, que los autores atribuyeron a una muestra demasiado homogénea (análisis post-hoc). Urzúa ${ }^{23}$ realizó un estudio en nuestro país en 345 niños hospitalizados con infección respiratoria baja (neumonía y bronquiolitis) que fueron evaluados con puntaje de tal por grupos.
Los resultados muestran una concordancia igualmente baja para sibilancias en el grupo de internos con poca experiencia $(\kappa=0,23)$ y residentes de pediatría con mayor experiencia $(\kappa=0,27)$. Estos estudios nos permiten concluir que la discriminación de ruidos respiratorios en tiempo real o a través de grabaciones no parece mejorar con el entrenamiento. De esta forma, sería deseable mantener un entrenamiento continuo independiente de los años de ejercicio.

El rendimiento de la auscultación pulmonar como herramienta diagnóstica de discriminación de enfermedad ha sido explorado en distintos escenarios con resultados variables. En un estudio realizado en urgencias para evaluar el rendimiento del examen físico en niños con fiebre se reporta baja sensibilidad (10-50\%) y alta especificidad (90-100) para diagnóstico de enfermedad bacteriana, entre ellas neumonía ${ }^{24}$. Por otra parte, la presencia de sibilancias en niños menores de 5 años con neumonía se ha reportado con un valor predictivo positivo de $96 \%$ para etiología viral ${ }^{25}$. Ante estos resultados, resulta inapropiado intentar generalizar el rendimiento diagnóstico de la auscultación pulmonar puesto que va a depender del escenario considerado. A modo de ejemplo, sirve considerar la importancia que se le da a la presencia de sibilancias como estándar de oro para el diagnóstico de asma cuando se reporta en forma recurrente, lo que se ha validado en forma repetida en distintas cohortes en todo el mundo ${ }^{26,27}$.

\section{Nuevos avances en auscultación pulmonar}

La auscultación pulmonar fue la única herramienta disponible para el diagnóstico de enfermedades respiratorias hasta la introducción de la radiografía de tórax en el siglo XX. Los avances en la tecnología y el análisis computacional de ruidos respiratorios han contribuido durante los últimos años a mejorar su rendimiento ${ }^{7}$. Los profesionales de la salud han confrontado la auscultación pulmonar enfocando principalmente los siguientes aspectos: mejorar nomenclatura, mejorar el entrenamiento de la técnica y aplicar nuevas tecnologías que mejoran la interpretación de los ruidos respiratorios.

Durante los últimos años se ha intentado mejorar el rendimiento de la auscultación tradicional por medio de un esfuerzo constante en alcanzar acuerdo en el lenguaje común. Con este objetivo las sociedades científicas han mejorado la nomenclatura de ruidos respiratorios que ha sufrido modificaciones con el tiempo ${ }^{20,28-30}$. Adicionalmente, la tecnología ha permitido comparar de forma objetiva ruidos respiratorios grabados que, como señal digital, permiten un análisis más profundo que han mejorado la interpretación de estos ${ }^{11,12,31}$. En las tablas 1 y 2 hemos resumido la nomenclatura actual 
recomendada y las características generales de los ruidos adventicios de la clasificación mencionada ${ }^{20}$.

En el entrenamiento de nuevos profesionales, es fundamental incentivar la técnica convencional de auscultación por medio del uso de tecnología audio visual y de simulación. Esto permite confrontar a profesionales con experiencias lo más reales y educativas posible que enriquecen su práctica ${ }^{31}$. Sin embargo, también la modernidad ha traído una peligrosa realidad que consiste en gran cantidad de información errónea. Una búsqueda reciente de ruidos respiratorios en supuestos sitios educativos realizada en la plataforma "YouTube" destaca la existencia de mucha información equívoca y confusa ${ }^{32}$. Es fundamental el esfuerzo que se pueda realizar a nivel educativo de las carreras de la salud para el entrenamiento básico de la técnica tradicional y eventualmente de nuevas tecnologías que se presentan como complementarias. Es deseable que estas herramientas estén también disponibles para profesionales con experiencia.
Tabla 1. Clasificación de los Ruidos respiratorios

I. Ruidos Respiratorios Normales
Ruido traqueal
Murmullo pulmonar
II. Ruidos por la transmisión de la voz
Broncofonía
Egofonía
Pectoriloquia
III. Ruidos adventicios o agregados
Continuos
Estridor
Sibilancias
Roncus
Discontinuos
Crepitaciones finas
Crepitaciones gruesas
Frotes pleurales

Tabla 2. Características de los ruidos respiratorios

\begin{tabular}{|c|c|c|c|c|}
\hline Nomenclatura & Características & Localización & Frecuencia & Enfermedades asociadas \\
\hline \multicolumn{5}{|l|}{ Ruidos normales } \\
\hline Ruido traqueal & $\begin{array}{l}\text { Timbre tubular, hueco. Inspiración y } \\
\text { espiración }\end{array}$ & $\begin{array}{l}\text { Escotadura esternal o región } \\
\text { cervical lateral }\end{array}$ & $100-5,000 \mathrm{~Hz}$ & \\
\hline $\begin{array}{l}\text { Murmullo } \\
\text { pulmonar }\end{array}$ & $\begin{array}{l}\text { Suave } \\
\text { Inspiración y fase inicial de espiración }\end{array}$ & Mayor parte del tórax & $100-1,000 \mathrm{~Hz}$ & \\
\hline \multicolumn{5}{|l|}{ Ruidos anormales } \\
\hline \multicolumn{5}{|l|}{ Continuos } \\
\hline Estridor & $\begin{array}{l}\text { Tono agudo de alta intensidad } \\
\text { Inspiración, puede ser bifásico (> } 250 \text { ms) }\end{array}$ & $\begin{array}{l}\text { Mayor en cuello, se irradia } \\
\text { a tórax }\end{array}$ & $>500 \mathrm{~Hz}$ & $\begin{array}{l}\text { Laringitis aguda } \\
\text { Laringomalacia } \\
\text { Epiglotitis } \\
\text { Anafilaxia } \\
\text { Parálisis cordal }\end{array}$ \\
\hline Sibilancias & $\begin{array}{l}\text { Tonalidad aguda } \\
\text { Espiración, puede ser bifásico (> } 80 \text { ms) }\end{array}$ & $\begin{array}{l}\text { Mayor parte del tórax } \\
\text { Se transmiten a la boca }\end{array}$ & $>400 \mathrm{~Hz}$ & $\begin{array}{l}\text { Asma y EPOC } \\
\text { Cuerpo extraño } \\
\text { Anillos vasculares } \\
\text { Tumores }\end{array}$ \\
\hline Roncus & $\begin{array}{l}\text { Tonalidad grave } \\
\text { Espiración, puede ser bifásico (> } 80 \text { ms) }\end{array}$ & Mayor parte del tórax & $\approx 150 \mathrm{~Hz}$ & $\begin{array}{l}\text { Asma } \\
\text { Bronquiolitis }\end{array}$ \\
\hline \multicolumn{5}{|l|}{ No continuos } \\
\hline $\begin{array}{l}\text { Crepitaciones } \\
\text { finas }\end{array}$ & $\begin{array}{l}\text { Ruido explosivo, corto ( } \approx 5 \text { ms) } \\
\text { Presente al final de inspiración }\end{array}$ & $\begin{array}{l}\text { Mayor parte del tórax } \\
\text { No se transmiten a la boca }\end{array}$ & $\approx 650 \mathrm{~Hz}$ & $\begin{array}{l}\text { Bronquiolitis } \\
\text { Neumonía } \\
\text { Fibrosis pulmonar }\end{array}$ \\
\hline $\begin{array}{l}\text { Crepitaciones } \\
\text { gruesas }\end{array}$ & $\begin{array}{l}\text { Ruido de estallido, alta intensidad } \\
\text { Presente ambas fases ( } \approx 15 \mathrm{~ms} \text { ) }\end{array}$ & $\begin{array}{l}\text { Mayor parte del tórax } \\
\text { Se transmite a la boca }\end{array}$ & $\approx 350 \mathrm{~Hz}$ & $\begin{array}{l}\text { Bronquiectasias } \\
\text { Bronquitis aguda } \\
\text { Traqueotomía }\end{array}$ \\
\hline Frote pleural & $\begin{array}{l}\text { Explosivo } \\
\text { Presente en ambas fases }\end{array}$ & $\begin{array}{l}\text { Bases pulmonares y región } \\
\text { axilar }\end{array}$ & $<350 \mathrm{~Hz}$ & $\begin{array}{l}\text { Pleuritis } \\
\text { Empema pleural }\end{array}$ \\
\hline
\end{tabular}


Existen hoy en día equipos que permiten grabar los ruidos respiratorios de un paciente con un dispositivo electrónico, para luego analizar las propiedades acústicas que caracterizan estos hallazgos denominado CORSA por sus siglas en inglés (Computerized respiratory sound analysis). Es una herramienta no invasiva, relativamente objetiva y estandarizada, que ayuda a caracterizar ruidos normales y adventicios (figuras 1,2 ), aunque todavía presenta gran interferencia de ruidos ambientales que no son fácilmente eliminados, como lo hace automáticamente el oído humano de forma inteligente ${ }^{33,34}$.

Los ruidos grabados son analizados y clasificados bajo diferentes métodos, que van desde la determinación empírica, hasta el uso de inteligencia artificial, método que ha suscitado gran interés y que se usa en la mayoría de los estudios ${ }^{35}$. Existe evidencia que ha mostrado que CORSA es más objetivo que el reporte de los padres o la auscultación de médicos entrenados ${ }^{33,35-37}$. Un meta-análisis reportó una sensibilidad de entre 70 a 95\% y una especificidad de 80 a 95\% para CORSA, dependiendo en gran medida de la intensidad del ruido ambiental que había en el momento de la grabación de los ruidos ${ }^{12}$. Un estudio reciente comparó los hallazgos entre análisis acústico y clínico mediante estetoscopio en pacientes en tiempo real y se logró discriminar adecuadamente entre distintos ruidos y mostrar una buena confiabilidad intra-evaluador ${ }^{6}$.

Esta técnica la implementaron en la pesquisa de sibilancias en 120 niños menores de 1 año, reportando una sensibilidad de $85,7 \%$ y $84,6 \%$ para sibilancias inspiratorias y espiratorias respectivamente, junto con una especificidad de $80,7 \%$ y $82,5 \%{ }^{10}$. En comparación, la evaluación por profesionales de la salud en dicho estudio mostró un nivel moderado de acuerdo inter-observador, demostrando que CORSA es una herramienta confiable que otorga información objetiva respecto a la presencia de sibilancias.

La aplicación del análisis de ruidos también abarca la caracterización de pacientes con distintas enfermedades que se presentan en forma similar. En esta línea, Sánchez ${ }^{38}$ reportó los resultados del análisis acústico en 76 lactantes con bronquiolitis y 34 prescolares con sibilancias recurrentes donde la información obtenida respecto del tipo de sibilancias que presenta cada paciente permitió su diferenciación clínica. El uso de esta tecnología ha permitido una interpretación más objetiva de los ruidos respiratorios, pero también ha tenido oportunidades de tener un rol en la toma de decisiones clínicas respecto a la terapia de pacientes con enfermedades respiratorias. Varios estudios han mostrado que CORSA ha sido útil en pacientes con asma en la detección de sibilancias durante las pruebas de provocación bronquial, contando con una fuerte correlación con el grado de obstrucción bronquial que presenta el paciente $^{39,40}$. En un estudio realizado en 22 lactantes con bronquiolitis aguda se pudo establecer la respuesta clínica al uso de salbutamol en relación al tipo de sibilancias que presentaba en el análisis acústico realizado ${ }^{41}$. Esto podría ser crucial por cuanto permite determinar en la práctica clínica pacientes que responden o no lo hacen a determinada terapia respiratoria.

Por último, también se ha explorado la utilidad que tendría el análisis de ruidos respiratorios en relación al control de la enfermedad que se estudia. La monitorización de sibilancias nocturnas utilizando CORSA en pacientes pediátricos y su correlación con actividad de enfermedad ha mostrado ser una herramienta no invasiva que posee buena asociación con otros parámetros convencionales utilizados para evaluar actividad de enfermedad (índices espirométricos, reactividad bronquial, reporte de síntomas diarios), y que pudiera asistir en la evaluación de eficacia de tratamiento de los pacientes $^{39,40,42,43}$.

En conclusión, se presenta en esta revisión un análisis crítico del rendimiento de la auscultación pulmonar pediátrica por medio del estudio de la concordancia entre profesionales y del análisis computacional de los ruidos respiratorios grabados con tecnología digital. Estos avances son promisorios y con toda probabilidad permitirán un mejor desarrollo de la auscultación objetiva abarcando el área diagnóstica y terapéutica de enfermedades respiratorias.

\section{Conflicto de intereses}

Los autores declaran no tener conflicto de intereses. 


\section{Referencias}

1. Laennec RTH. De l'asucultation médiate ou traité du diagnostic des maladies des poumons e tud couer. Paris: Brosson \& Chaudé, 1819.

2. Bishop PJ. Evolution of the stethoscope. J R Soc Med. 1980;3:448-56.

3. Markel H. The Stethoscope and the Art of Listening. N Engl J Med. 2006;354:551-53.

4. Sarkar H, Madabhavi I, Niranjan N, et al. Auscultation of respiratory system. Ann Thorac Med 2015;10:158-68.

5. Brooks D, Thomas J. Interrater Reliability of Auscultation of Breath Sounds Among Physical Therapists. Phys Ther. 1995; 75:1082-88.

6. Elphick HE, Lancaster GA, Solis A, et al. Validity and reliability of acoustic analysis of respiratory sounds in infants. Arch Dis Child. 2004; 89:1059-63.

7. Melbye H, Garcia-Marcos L, Brand P, et al. Wheezes, crackles and rhonci: simplifying description of lung sounds increases the agreement on their classification: a study of 12 physicians' classification of lung sounds from video recording. BMJ Open Respiratory Research. 2016;3:e000136.

8. Gajdos V, Beydon N, Bommenel L, et al. Inter-observer agreement between physician, nurses, and respiratory therapist for respiratory clinical evaluation in bronchiolitis. Pediatr Pulmonol. 2009;44:754-62.

9. Muñoz J, Saavedra C, Adasme R, Arellano D, Sánchez I, Bertrand P. Concordance in Discriminating Recordings of Different Lung Sounds Between Physiotherapists. Respiratory Care 2020;65(2):177-82.

10. Puder LC, Fischer HS, Wilitzki S, Usemann J, Godfrey S, Schmalisch G. Validation of computerized wheeze detection in young infants during the first months of life. BMC Pediatr. 2014;14:257.

11. Oliveira A, Marques A. Respiratory sounds in healthy people: A systematic review. Respir Med. 2014;108:550-70.

12. Gurung A, Scrafford C, Tielsch J, et al. Computerized Lung Sound Analysis as diagnostic aid for the detection of abnormal lung sounds: a systematic review and meta-analysis. Respir Med. 2011;105:1396-403.

13. Murphy R. In defense of the stethoscope. Respir Care. 2008;53:355-69.

14. Taussig L, Landau L. Pediatric Respiratory Medicine. Second ed. Philadelphia: Elsevier; 2008.

15. Wilmott R, Bush A, Deterding R, Ratjen F. Kending's Disorders of the Respiratory Tract in Children. 9th ed. Philadelphia: Elsevier; 2018.

16. Zorc J, Alpern E, Brown LW, Loomes KM, Marino BS, Mollen CJ. Schwartz's
Clinical Handbook of Pediatrics. 5th ed. Philadelphia: Lippincott Williams \& Wilkins; 2012.

17. Margolis P, Ferkol T, Marsocci S, et al Accuracy of the clinical examination in detecting hypoxemia in infants with respiratory illness. J Pediatr. 1994;124:552-60.

18. Bekhof J, Reimink R, Bartels I, et al. Large observer variation of clinical assessment of dyspnoeic wheezing children. Arch Dis Child. 2015; 100:649-53.

19. Jensen E, Panitch H, Feng R, et al. Interobserver Reliability of the Respiratory Physical Examination in Premature Infants: A Multicenter Study. J Pediatr. 2016; 178:87-92.

20 Bohadana A, Izbicki G, Kraman SS. Fundamentals of Lung Auscultation. N Engl J Med. 2014;370:744-51.

21. Kiyokawa H, Greenber M, Shirota K, et al. Auditory detection of simulated crackles in breath sounds. Chest. 2001;119:188692.

22. Allingame S, Williams T, Jenkins $S$, et al. Accuracy and reliability of physiotherapists in the interpretation of tape-recorded lung sounds. Aust J Physiother. 1995;41:179-84.

23. Urzua S, Duffau G, Zepeda G, Sagredo S. A study of clinical concordance in pre and postgraduate students in Paedriatrics: Tal score. Rev Chil Pediatr. 2002; 73(5):471-7.

24. Craig JC, Williams GJ, Jones M, et al. The accuracy of clinical symptoms and signs for the diagnosis of serious bacterial infection in young febrile children: prospective cohort study of 15781 febrile illnesse. BMJ 2010;340: c1594.

25. Nascimento-Carvalho AC, Ruuskanen O, Nascimento-Carvalho CM. Wheezing independently predicts viral infection in children with community-acquired pneumonia. Pediatr Pulmonol. 2019;54(7):1022-8.

26. Hay AD, Heron J, Ness A. The prevalence of symptoms and consultations in preschool children in the Avon Longitudinal Study of Parents and Children (ALSPAC): a prospective cohort study. Fam Pract. 2005;22(4):367-74.

27. Taussig LM, Wright AL, Holberg CJ, Halonen M, Morgan WJ, Martinez FD. Tucson Children's Respiratory Study: 1980 to present. J Allergy Clin Immunol. 2003;111(4):661-75.

28. Mikami R, Murao M, Cugell D, et al. International symposium on lung sounds: Synopsis of proceedings. Chest. 1987;92:342-45.

29. Cruz Mena E. Lung sound nomenclature, Am Rev Respir Dis. 1988;137:986.

30. Pasterkamp H, Brand P, Everard M, et al. Towards the standardization of lung sound nomenclature. Eur Respir J. 2016; 47:724-32.

31. Ellington L, Emmanouilidou D, Elhilali $\mathrm{M}$, et al. Developing a Reference of Normal Lung Sounds in Healthy Peruvian Children. Lung. 2014; 92:765-73.

31. Sanchez I. Atlas de Ruidos Respiratorios, Facultad de Medicina, Universidad Católica de Chile. 2004. http:// publicacionesmedicina.uc.cl/AtlasRuidos/ Default.html

32. Sunderland N, Camm C, Glover K, et al. A quality assessment of respiratory auscultation material on YouTube. Clin Med (Lond) 2014;14:391-5.

33. Prodhan P, De la Rosa R, Shubina M, et al. Wheeze detection in the pediatric intensive care unit: comparison among physician, nurses, respiratory therapists, and a computerized respiratory sound monitor. Respir Care. 2008; 53:1304-9.

34. Marques A, Oliveira A, Jácome C. Computerized adventitious respiratory sounds as outcome measures for respiratory therapy: a systematic review. Respir Care. 2014;59:765-76.

35. Sovijarvi A, Malmberg L, Charbonneau $\mathrm{G}$, et al. Characteristics of breath sounds and adventitious respiratory sounds. Eur Respir Rev. 2000;10:591-96.

36. Levy M, Godfrey S, Irving C, et al. Wheeze detection: recordings vs. assessment of physician and parent. J Asthma. 2004; 41:845-53.

37. Elphick H, Ritson S, Rodgers $\mathrm{H}$, et al. When a "wheeze" is not a wheeze: acoustic analysis of breath sounds in infants. Eur Respir J. 2000;16:593-97.

38. Sánchez I., Navarro H., Bertrand P.,et al. Acoustic analysis of wheezing in infants with acute bronchial obstruction: A follow-up study. Rev Med Chile 2002;130:760-39.

39. Fischer H, Puder L, Wilitzki S, et al. Relationship between computerized wheeze detection and lung function parameters in young infants. Pediatr Pulmonol. 2016;51:402-10.

40. Fiz J, Jane R, Izquierdo J, et al. Analysis of forced wheezes in asthma patients. Respiration. 2006;73:55-60.

41. Sánchez I, Vizcaya C, García D, Campos E. Response to bronchodilator in infants with bronchiolitis can be predicted from wheeze characteristics. Respirology. 2005;10:603-8.

42. Bentur L, Beck R, Shinawi M,et al. Wheeze monitoring in children for assessment of nocturnal asthma and response to therapy. Eur Respir J. 2003; 21:621-6.

43. Boner A, Piacentini G, Peroni D, et al. Children with nocturnal asthma wheeze intermittently during sleep. J Asthma. 2010; 47:290-94. 